
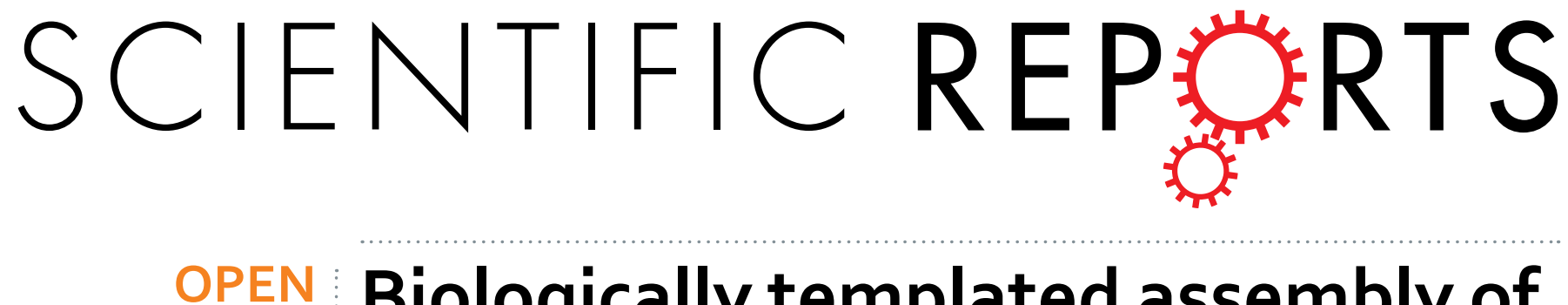

\title{
Biologically templated assembly of hybrid semiconducting nanomesh for high performance field effect transistors and sensors
}

Received: 07 July 2016

Accepted: 03 October 2016

Published: 20 October 2016

\author{
Hye-Hyeon Byeon ${ }^{1,2, *}$, Seung-Woo Lee ${ }^{1,+, *}$, Eun-Hee Lee ${ }^{3}$, Woong Kim ${ }^{4}$ \& Hyunjung Yi ${ }^{1}$
}

Delicately assembled composites of semiconducting nanomaterials and biological materials provide an attractive interface for emerging applications, such as chemical/biological sensors, wearable health monitoring devices, and therapeutic agent releasing devices. The nanostructure of composites as a channel and a sensing material plays a critical role in the performance of field effect transistors (FETs). Therefore, it is highly desirable to prepare elaborate composite that can allow the fabrication of high performance FETs and also provide high sensitivity and selectivity in detecting specific chemical/ biological targets. In this work, we demonstrate that high performance FETs can be fabricated with a hydrodynamically assembled composite, a semiconducting nanomesh, of semiconducting singlewalled carbon nanotubes (S-SWNTs) and a genetically engineered M13 phage to show strong binding affinity toward SWNTs. The semiconducting nanomesh enables a high on/off ratio $\left(\sim 10^{4}\right)$ of FETs. We also show that the threshold voltage and the channel current of the nanomesh FETs are sensitive to the change of the M13 phage surface charge. This biological gate effect of the phage enables the detection of biologically important molecules such as dopamine and bisphenol A using nanomesh-based FETs. Our results provide a new insight for the preparation of composite material platform for highly controllable bio/electronics interfaces.

Integration of semiconducting nanomaterials with biological materials has been extensively investigated in various fields including bio-sensors, brain science, cancer research, tissue engineering, and drug delivery ${ }^{1-5}$. The integration has been motivated and driven by the advantages of the semiconducting nanomaterial-based devices that are sensitive to small amount of chemical or biological targets and/or the nanostructures that provide large effective surface area for maximal interaction with ionic environment ${ }^{6-9}$. However, the device performance strongly depends on the dispersion state of individual materials in the nano/bio composites and the amount of defects generated during the fabrication process ${ }^{10-12}$. Therefore, the challenge is to prepare the nano-bio composite materials in which each nanomaterial is effectively separated from one another without being agglomerated while intrinsic properties of the nanomaterials and the biomaterials are well-preserved during the dispersion process which would contribute to maximizing device performance ${ }^{13,14}$.

Molecular recognition of biological materials not only enables selective binding to target chemical or biological species but also offers a non-destructive means to precisely control the interactions between biomolecules and non-biological materials ${ }^{15-18}$. Various biomolecules such as DNA, peptides, and proteins have been utilized to functionalize surfaces of electronic materials or to stabilize nanoscale electronic materials in aqueous solution without deteriorating their electronic properties ${ }^{19-22}$. For example, specific short peptide sequences have been identified through affinity-based selection processes such as bio-panning and have been utilized for binding of various electronic materials ${ }^{17,23-26}$. In addition, biological materials inherently possess various chemical

${ }^{1}$ Post-Silicon Semiconductor Institute, Korea Institute of Science and Technology, Seoul, 02792, Republic of Korea. ${ }^{2}$ Department of Nano Semiconductor Engineering, Korea University, Seoul, 02841, Republic of Korea. ${ }^{3}$ Department of Environmental Science and Engineering, Ewha Womans University, Seoul, 03760, Republic of Korea. ${ }^{4}$ Department of Materials Science and Engineering, Korea University, Seoul, 02841, Republic of Korea. ${ }^{\dagger}$ Present address: Department of Fine Chemistry, Seoul, National University of Science and Technology, Seoul, 01811, Republic of Korea. *These authors contributed equally to this work. Correspondence and requests for materials should be addressed to W.K. (email: woongkim@korea.ac.kr) or H.Y. (email: hjungyi@kist.re.kr) 
functional groups. Therefore, a biological template material encoded with specific binding affinity toward nanoscale electronic materials could serve as an ideal platform to assemble nanoscale semiconducting material and at the same time confer additional functionality for highly controllable electrochemical interfaces.

Recently, it has been demonstrated that a filamentous M13 phage showing strong binding affinity to the surface of single-walled carbon nanotubes (SWNTs) could effectively disperse unsorted SWNTs and can be used to assemble a conductive nanomesh via a hydrodynamic assembly process ${ }^{27,28}$. An M13 phage is a filamentous biological material with a diameter of $\sim 6.5 \mathrm{~nm}$ and a length of $\sim 880 \mathrm{~nm}$, and the capsid proteins at various locations of the M13 phage can be genetically engineered to include specific peptide sequences that bind targeted materials ${ }^{29-31}$. Owing to its filamentous shape, the M13 phage has been shown to effectively interact with one-dimensional nanomaterials such as SWNTs ${ }^{32}$. The assembled conductive nanomesh had fine nanostructures, high conductivity and good adhesion with various substrates including flexible substrates. As a result, the conductive nanomesh-integrated flexible neural probes drastically decreased the in vivo contact impedance between bio-fluids and the neural probes, and significantly improved the detection rate of biologically important but weak brain signals of mouse ${ }^{27}$. However, applications of the conductive nanomesh are limited due to very low on/off current ratio $\left(\mathrm{I}_{\mathrm{on}} / \mathrm{I}_{\mathrm{off}} \sim 10\right)$; successful assembly of semiconducting nanomesh would greatly extend the scope of applications towards the fabrication high performance transistors and biological sensors.

Here, we demonstrate that high quality semiconducting nanomesh can be fabricated based on the genetically engineered filamentous biological template, M13 phage, and semiconducting-enriched SWNTs (S-SWNTs). We also report a biological gate effect of the semiconducting nanomesh-based FETs. The semiconducting nanomesh can be prepared in macroscale ( several centimeters) and an array of nanomesh-based electronic devices can be fabricated without relying on lithographic, chemical or annealing process. Owing to the excellent dispersion of the S-SWNTs, nanomesh FETs show high on/off ratio of $10^{4}$. Importantly, the semiconducting nanomesh-based FETs are sensitive to the change of the local charge density and $\mathrm{pH}$ via the biological gate effect of the phage. This charge sensitive nature allows the semiconducting nanomesh-based electrolyte-gated FETs (e-FETs) to detect important biological chemicals such as dopamine and bisphenol A. We envision that the biologically integrated semiconducting nanomesh would serve as a new material platform for highly controllable bio/electronics interfaces and flexible biosensors.

\section{Results}

Biologically templated assembly of semiconducting nanomesh. Figure 1a schematically illustrates the biological template material, p8GB\#1 M13 phage containing on its surface (p8) a specific peptide sequence with strong affinity for SWNTs, to bind and to align S-SWNTs (Fig. 1b) along the length of its body. The peptide sequence of the p8GB\#1 M13 phage was identified by applying a bio-panning process using a phage display $\mathrm{p} 8$ peptide library that was constructed in our laboratory ${ }^{28}$. The hydrophobicity pattern and the aromatic amino acid tryptophan (W) of p8GB\#1 are expected to be mainly responsible for the interaction with S-SWNTs ${ }^{27}$. The surface peptide of the p8GB\# 1 phage has also various charged functional groups, including acidic $(\mathrm{D}$, carboxyl group, $\left.\mathrm{pK}_{\mathrm{a}} \sim 3.9\right)$ and basic $\left(\mathrm{K}\right.$, amine, $\left.\mathrm{pK}_{\mathrm{a}} \sim 10.54\right)$ residues, as shown in Fig. 1a and Fig. S1. The net surface charge of the phage produces an effective electric field in the electrolyte solution according to Poisson equation ${ }^{33}$. The net surface charge density can be inferred from the zeta potential, and the measured zeta potential of the p8GB\#1 phage is presented in Fig. 1c. Since the $\mathrm{pK}_{\mathrm{a}}$ of the side chain functional group of $\mathrm{D}$ is lower than that of $\mathrm{K}$, the change of the net surface charge was dominated by deprotonation of the carboxyl group as the $\mathrm{pH}$ was increased within the tested $\mathrm{pH}$ range. Since potential (electric field) is directly related to the surface charge density and the M13 phage is directly interfacing with the semiconducting SWNT- channel, the M13 phage can behave as local gate that is sensitive to local charge density via change of $\mathrm{pH}$ or binding of small molecules, as schematically illustrated in Fig. 1d and demonstrated further below ${ }^{33}$.

Figure 2a schematically illustrates the process to assemble semiconducting nanomesh. The hydrodynamic assembly works based on a concentration polarization effect ${ }^{27}$. Concentration polarization is a diffusion-based hydrodynamic phenomenon in which the concentration of colloidal particles such as S-SWNTs and the M13 phage that are bigger than the molecular-weight cut-off (MWCO) of the dialysis membrane become enriched whereas species smaller than the MWCO of the membrane such as surfactant molecules and ions become depleted near the inside boundary of the dialysis membrane. When surfactant-dispersed S-SWNTs were dialyzed in the presence of p8GB\#1 phage, p8GB\#1 phage bound S-SWNTs and the p8GB\#1-bound S-SWNTs were assembled into a nanostructured network, i.e., a nanomesh around the dialysis membrane. Here, it is noted that the sorted S-SWNTs were surfactant-exchanged with sodium cholate before the hydrodynamic process for the stable assembly of the nanomesh. Removing the dialysis membrane after the dialysis readily produced a large freestanding nanomesh film in solution as shown Fig. 2 a. In contrast, in the absence of $\mathrm{p} 8 \mathrm{~GB} \# 1$, S-SWNTs formed fragile films or aggregates and did not form large stable films (Fig. S2). Moreover, a different M13 phage clone that did not have binding affinity toward S-SWNTs also only produced aggregates or fragmented SWNTs (Fig. S3). These results imply that the binding affinity of p8GB\#1 phage is critical for the formation of the nanomesh. It also suggests that sorted S-SWNTs can be successfully assembled into a nanomesh using the hydrodynamic assembly method via surfactant exchange process. The thickness of the semiconducting nanomesh film produced in the presence of p8GB\#1 was about $\sim 100 \mathrm{~nm}$ and the size was $\sim 100 \mathrm{~cm}^{2}$. The size of the nanomesh, being dependent on the size of the dialysis membrane, could be in principle further scaled up by using larger dialysis membranes. The isolated freestanding nanomesh was readily transferred onto various substrates without chemical etching as shown in Fig. 2a. The transmittance curve of the nanomesh transferred onto a transparent substrate is shown in the Fig. 2b. The molar ratio is S-SWNT:p8GB\#1=1:2. Here, it is noted that although the size of the M13 phage is much bigger than the S-SWNTs, allowing binding of multiple SWNTs per M13 phage, a compatible molar ratio of the M13 phage to the S-SWNTs was used due to the slower diffusion of the M13 phage than the SWNTs during the nanomesh assembly ${ }^{27}$. The absorption peaks in the wavelength range of $400 \mathrm{~nm} \sim 600 \mathrm{~nm}$ correspond to the optical transition peaks from the S-SWNTs and the large absorption peak around $270 \mathrm{~nm}$ is due to the M13 phage ${ }^{30,32}$. 
(a)

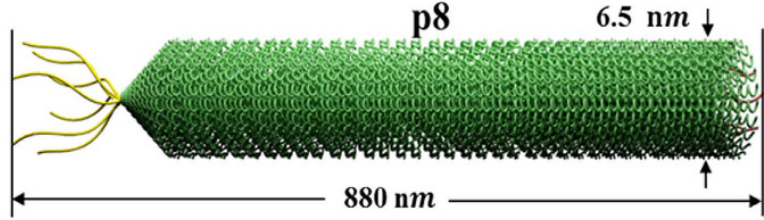

p8 peptide sequence : N'-ADSWAADIPDPAKAA...

- DSWAADIP : binding affinity towards SWNTs

- D and $\mathbf{K}$ : biological gate effect on semiconductors

- $\mathbf{N}$ ' and $\mathbf{K}$ : conjugation sites for biomolecules (b)

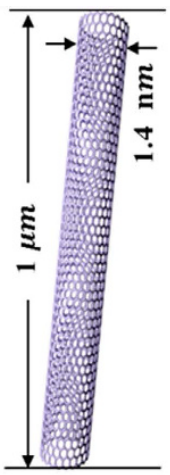

Semiconducting SWNTs

(S-SWNTs) (c)

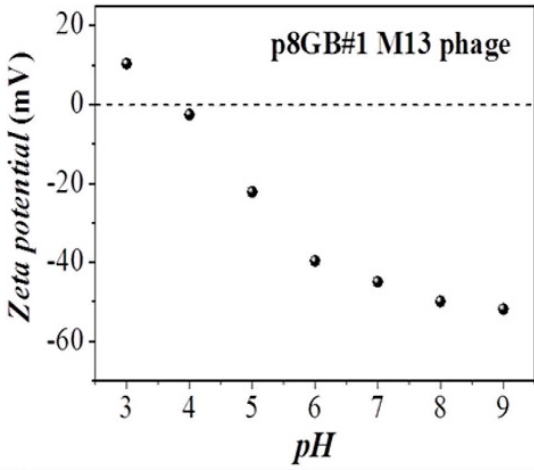

(d)

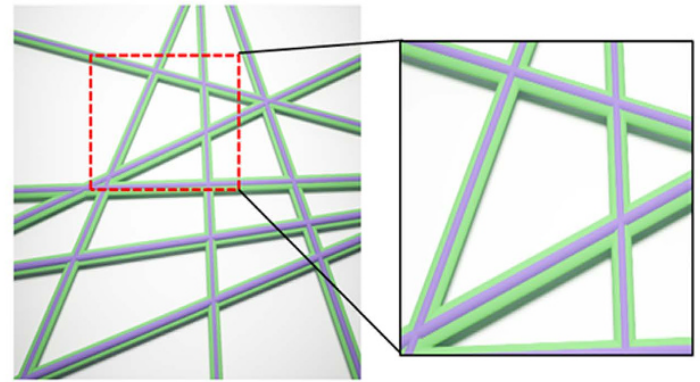

Figure 1. Components and the nanostructure of the semiconducting nanomesh. (a) A schematic of the biological template material, p8GB\#1 M13 phage and (b) the semiconducting SWNT (S-SWNT). The phage contains a specific peptide sequence for binding SWNTs expressed on its body surface (p8). The body surface peptides also function as a biological gate on S-SWNTs and provide conjugation sites for additional biomolecules. (c) Zeta potentials of the $\mathrm{p} 8 \mathrm{~GB} \# 1$ phage at various $\mathrm{pH}$ values. (d) A schematic of the nanostructure of the semiconducting nanomesh. The M13 phage also serves as a biological gate on the intimately bound S-SWNTs via the charged surface peptides.

The non-destructive nature of the biological template-based SWNT-assembly and the transfer process was confirmed using Raman spectroscopy ${ }^{34}$. The Raman spectrum of the nanomesh transferred onto a $\mathrm{SiO}_{2} / \mathrm{Si}_{\text {sub- }}$ strate is shown and compared with the one from the as-received S-SWNTs in Fig. 2c. The nanomesh yielded a radial breathing mode (RBM) peak that is a characteristic of carbon nanotubes, the graphitic peak $(G)$, and a defect-related peak $\left(\mathrm{D}, \sim 1,400 \mathrm{~cm}^{-1}\right)$ in the spectrum. The negligible intensity of the $\mathrm{D}$ peak and the similarity of this Raman spectrum to that from the as-received S-SWNTs confirmed the non-destructive nature of the assembly and the transfer process. The nanostructure and the binding scheme of the assembled semiconducting nanomesh were examined using transmission electron microscopy (TEM). The transmission electron micrograph and the energy-filtered (EF) mapping of the corresponding region of the semiconducting nanomesh are shown in Fig. 2d-f. In general, SWNTs produce sharp and high-contrast lines whereas the biological materials such as M13 phage show dark and diffused lines. The transmission electron micrograph of the semiconducting nanomesh shows sharp lines (indicated by yellow arrows) overlapped with dark and diffuse lines (indicated by white arrows), suggesting the p8GB\#1 phage bound S-SWNTs along the body lengths in the nanomesh. To further confirm the binding scheme of the semiconducting nanomesh, an element specific TEM mapping was conducted. The carbon and the nitrogen mapping images of the corresponding TEM image are shown in Fig. 2e,f, respectively. Since S-SWNTs and surfactants do not have nitrogen elements whereas the p8GB\#1 M13 phage contains nitrogen components coming from the peptides, the element specific TEM confirms that the p8GB\#1 phage bound the S-SWNTs along its body length within the nanomesh matrix, as schematically illustrated in Fig. 1d. This binding scheme is similar to the conductive nanomesh assembled using mixed SWNTs ${ }^{27}$. However, when using the semiconducting-enriched SWNTs, this unique binding scheme of the hybrid nanomesh and the non-destructive assembly process additionally enabled biological gating by the phage on the semiconducting SWNTs, as demonstrated further below.

Semiconducting nanomesh-based FETs. The electrical transport properties of the semiconducting nanomesh as an FET channel have been investigated by controlling the molar ratio of S-SWNT:p8GB\#1 to produce a high $\mathrm{I}_{\mathrm{on}} / \mathrm{I}_{\text {off }}$ of macroscale field-effect transistors. The processes to fabricate the FETs are schematically illustrated in Fig. 3a. The scanning electron micrographs of a representative FET device and the channel region 
(a)
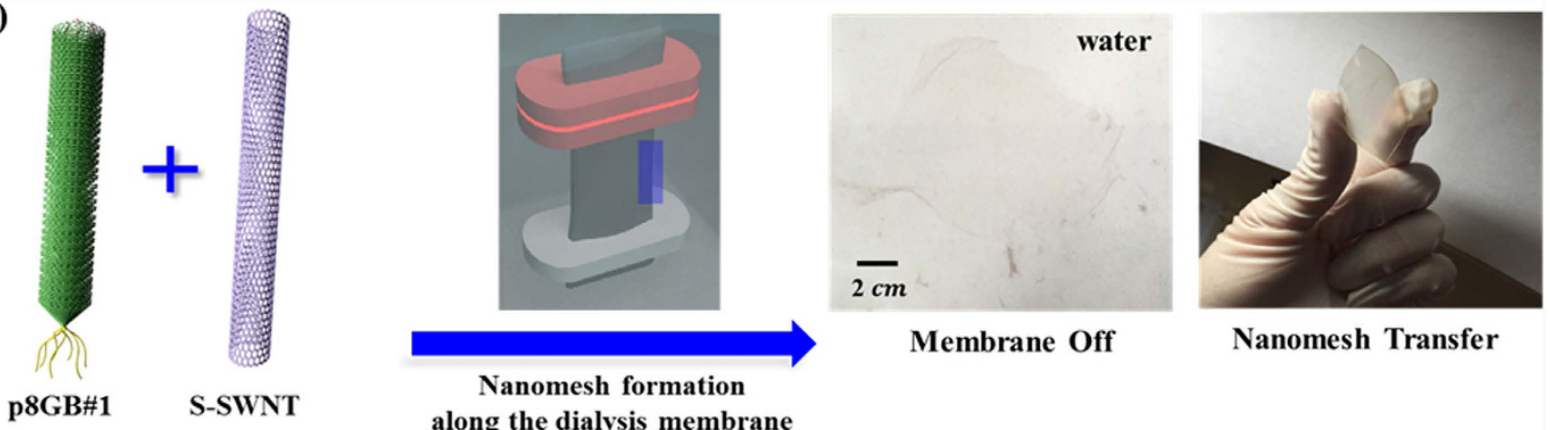

Membrane Off

Nanomesh Transfer

Nanomesh formation along the dialysis membrane

(b)

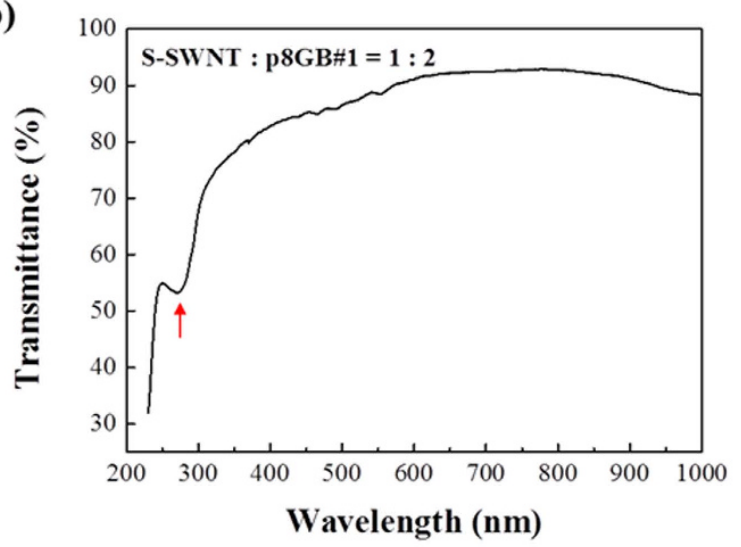

(c)

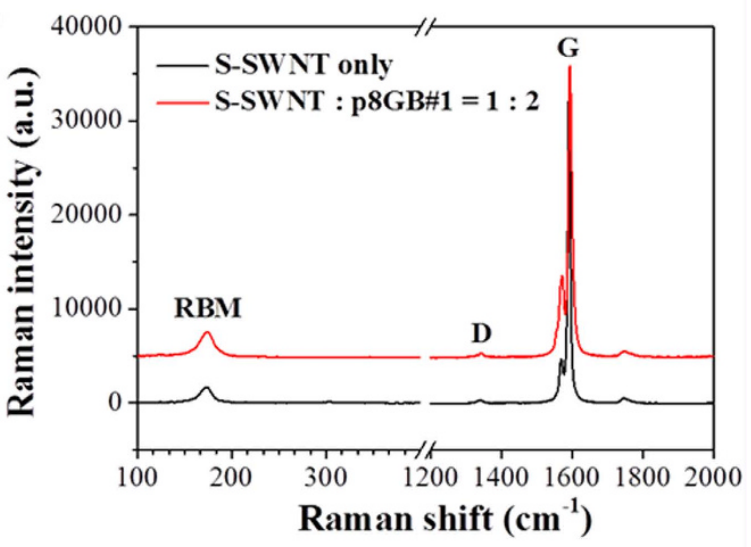

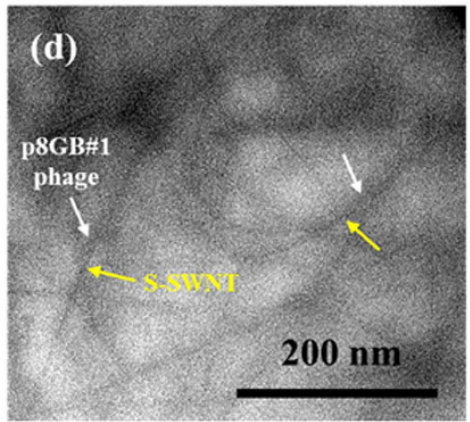
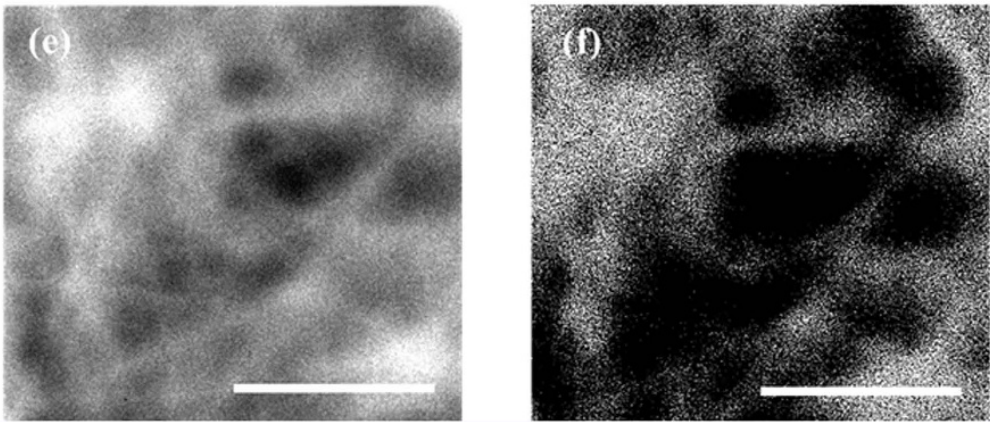

Figure 2. Fabrication processes and characteristics of the semiconducting nanomesh. (a) Processes to assemble hybrid semiconducting nanomesh using a biological template material in solution and the processes to provide the semiconducting nanomesh on arbitrary substrates. The semiconducting nanomesh transferred onto a transparent flexible substrate (PES) is shown. (b) The transmittance curve of the nanomesh. The molar ratio of S-SWNT:p8GB\#1 = 1:2. The absorption peaks in the wavelength range of $400 \mathrm{~nm} \sim 600 \mathrm{~nm}$ correspond to the optical transition peaks from S-SWNTs and the large absorption peak around $270 \mathrm{~nm}$ is due to the M13 phage ${ }^{30,32}$. (c) Raman spectrum of the hybrid semiconducting nanomesh on a $\mathrm{SiO}_{2} / \mathrm{Si}$ substrate compared with the solution-dispersed S-SWNTs drop-casted on $\mathrm{SiO}_{2} / \mathrm{Si}$ substrate (S-SWNT-only). (d) High-magnification transmission electron micrograph of the assembled hybrid semiconducting nanomesh. The sharp and highcontrast lines (indicated by yellow arrows) in the image correspond to the S-SWNTs whereas the dark and diffuse lines (indicated by white arrows) overlapping with the S-SWNTs are from the p8GB\#1 phage. (e) Carbon mapping image. (f) Nitrogen mapping image. Since the M13 phage contains both carbon and nitrogen whereas SWNTs possess carbon only, the nitrogen mapping indicates the locations of the M13 phage.

are shown in Fig. 3b. The transfer characteristics (the source-drain current, $\mathrm{I}_{\mathrm{DS}}$, versus the gate voltage, $\mathrm{V}_{\mathrm{G}}$ ) and the output characteristics ( $I_{D S}$ vs. the source-drain voltage, $V_{D S}$ ) from the nanomeshes of different molar ratios are presented in Fig. 4a and Fig. S4. These curves show that as the molar ratio of the phage was increased, the overall $\mathrm{I}_{\text {on }} / \mathrm{I}_{\text {off }}$ drastically improved. Here, the $\mathrm{I}_{\text {on }} / \mathrm{I}_{\text {off }}$ was defined as the difference between the maximum and the minimum $\mathrm{I}_{\mathrm{DS}}$ for an applied $\mathrm{V}_{\mathrm{DS}}$. The increased $\mathrm{I}_{\mathrm{on}} / \mathrm{I}_{\text {off }}$ with increasing molar ratio of $\mathrm{p} 8 \mathrm{~GB} \# 1$ is mainly due to the decreased $\mathrm{I}_{\text {off. }}$. The $\mathrm{I}_{\text {off }}$ is generally related to the metallic SWNTs ${ }^{10}$. Although we used $99 \%$ semiconducting enriched SWNTs, there still exist metallic SWNTs. In addition, the nanomesh with the higher molar ratio of the phage showed more effectively de-bundled networks (Fig. S5). Aggregated S-SWNTs could transfer electrons between tubes ${ }^{32}$. When the bandgap of S-SWNTs is small (when the diameter of SWNTs is large) and 
(a)
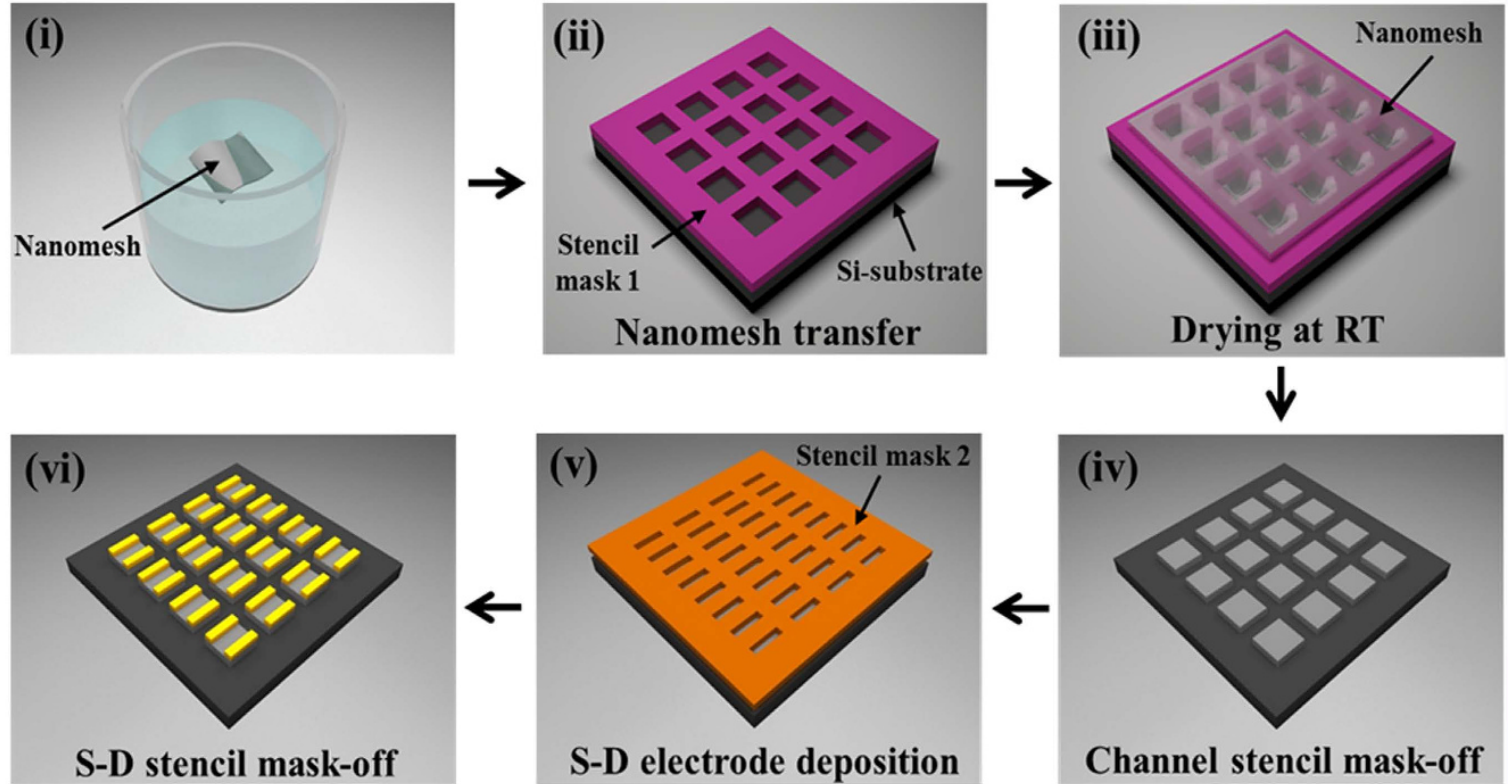

(b)

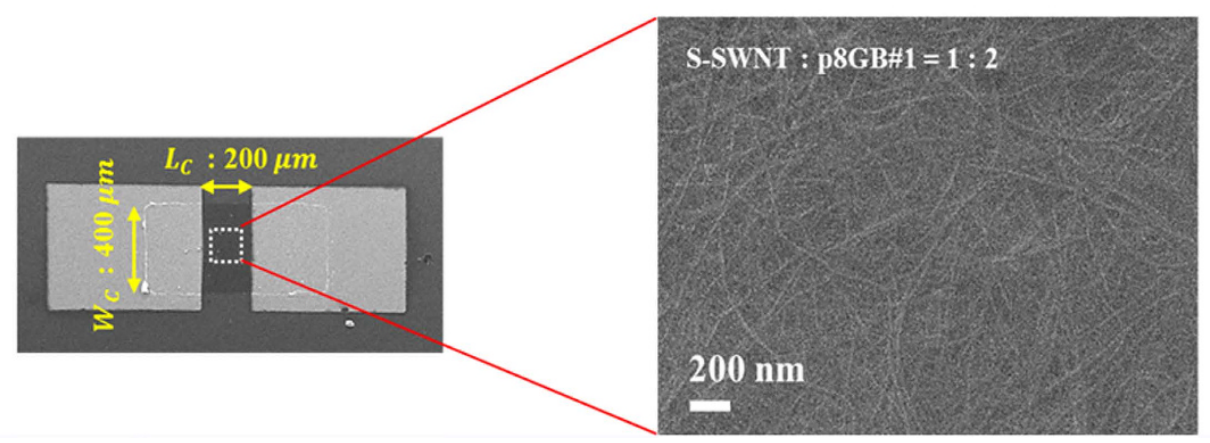

Figure 3. Non-destructive fabrication of semiconducting nanomesh-based FETs. (a) Processes used to fabricate the semiconducting nanomesh-based back-gated FET. (b) The scanning electron micrographs of a representative back-gated FET device and the channel region. The molar ratio of S-SWNT:p8GB\#1 = 1:2.

the heterogeneity of dimensions of SWNTs is large, the aggregated S-SWNTs could behave as metallic ones,

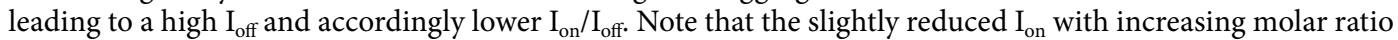
of the phage is due to the electrically insulating nature of the phage. Therefore, these results suggest that the p8GB\#1 phage improved the overall device performance by preventing S-SWNTs from bundling and by providing well-controlled nanostructures over a large area.

The output characteristics and the transfer characteristics of a representative back-gated FET from the 1:2 nanomesh are shown in Fig. 4 b,c. The best $\mathrm{I}_{\text {on }} / \mathrm{I}_{\text {off }}$ was found to be $\sim 1.34 \times 10^{4}$ at $\mathrm{V}_{\mathrm{DS}} \sim 0.1 \mathrm{~V}$. The $\mathrm{I}_{\text {on }}$ and $\mathrm{I}_{\text {off }}$ values were observed to be $1.61 \times 10^{-8} \mathrm{~A}$ and $1.20 \times 10^{-12} \mathrm{~A}$, respectively. The threshold voltage, $\mathrm{V}_{\text {th }}$, was $-23.83 \mathrm{~V}$, with a hole mobility, $\mu_{\mathrm{h}}$, of $0.17 \mathrm{~cm}^{2} / \mathrm{V} \mathrm{s}$ at $\mathrm{V}_{\mathrm{DS}}=0.1 \mathrm{~V}$ (supplementary equation). The $\mathrm{I}_{\mathrm{on}} / \mathrm{I}_{\mathrm{off}}$ ratio of the semiconducting nanomesh is much higher than that of the nanomesh from mixed SWNTs by three orders of magnitude ${ }^{27}$. Although this value is lower than the reported highest performance from SWNT-based FETs realized using other approaches, the performance of the nanomesh-based FET could be still compatible to those from high-performance SWNT-FETs as summarized in Fig. S6. These results suggest that the biologically templated assembly approach can provide macroscale high-performance SWNT-based FETs at a potentially large scale under ambient conditions in a simple and non-destructive manner.

Note that the transfer curve of the nanomesh showed anticlockwise hysteresis as indicated by the arrows of Fig. 4c. The degree of hysteresis, $\Delta \mathrm{V}_{\mathrm{HYST}}$, defined as the voltage width at the half-maximum of $\mathrm{I}_{\mathrm{DS}}$, increased with increasing molar ratio of the phage as shown in Fig. $4 \mathrm{~d}$. It is nown that the water molecules and the silanol groups on the surface of the $\mathrm{SiO}_{2}$ back-gate oxide are largely responsible for the hysteresis of the back-gated CNT FET $^{35-37}$. Due to the many hydrophilic amino acid residues (D, S, and K) on the surface of the p8GB\#1 phage body (Fig. 1a and Fig. S1), the increasing hysteresis of the semiconducting nanomesh was ascribed to the increased hydrophilicity of the assembled nanomesh. However, this hysteresis became negligible under an electrolyte gated configuration as demonstrated below. This could be ascribed to the back-gate dielectric, which is mainly responsible for hysteresis, not having been involved in the electrolyte gated configuration ${ }^{38,39}$. 

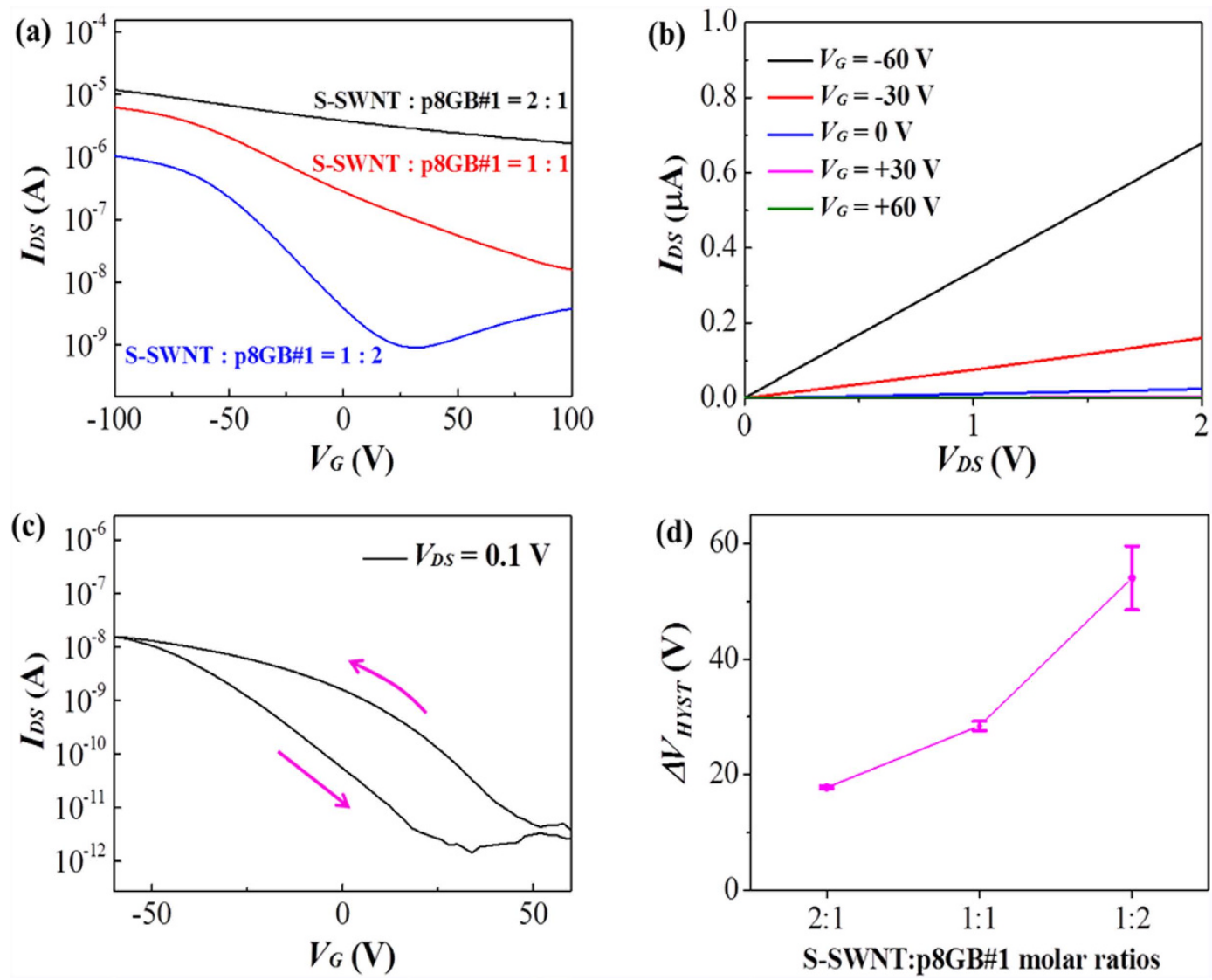

Figure 4. Characteristics of the semiconducting nanomesh-based FETs. (a) The effect of the phage on the transfer characteristics of the semiconducting nanomesh-based FETs. The (b) output characteristics and (c) transfer characteristics of the nanomesh-based FET with the molar ratio of 1:2. (d) The effect of the phage on the hysteresis of back-gated FETs. The $\Delta \mathrm{V}_{\mathrm{HYST}}$ is defined as the voltage width at the half-maximum of $\mathrm{I}_{\mathrm{DS}}$. Each data point is the mean \pm s.d. from $\mathrm{n}=3$ devices.

Biological gate effect of the semiconducting nanomesh-based e-FETs. The biological template approach enables an intimate contact between the chemical functional groups of the phage and the semiconducting SWNTs along their lengths. To investigate the gate effect of the phage template on the semiconducting channel, at first the transfer curves at different $\mathrm{pH}$ values were examined under an e-FET configuration, as schematically shown in Fig. $5 \mathrm{a}^{40}$. For the e-FET measurement, the source, drain and the water-based electrolyte gate (WG) potential were driven in a biological liquid electrolyte (10 mM PBS) using a bipotentiostat system (Autolab, PGSTAT $128 \mathrm{~N}$ ). The source and drain electrodes were designated as two working electrodes (WE1 and WE2), the $\mathrm{Ag} / \mathrm{AgCl}$ water-based electrolyte gate $(\mathrm{WG})$ as reference electrode ( $2 \mathrm{~mm}$ dia. filled with $3 \mathrm{M} \mathrm{KCl})$, and the additional Pt wire as the counter electrode (CE). The output characteristics of the e-FET are shown in Fig. S7. The transfer characteristics of the e-FET measured at various $\mathrm{pH}$ conditions are shown in Fig. 5b. Here, it is noted the $\mathrm{V}_{\text {th }}$ value of the e-FET was found to be less than $1 \mathrm{~V}$, which is significantly lower than the value from the back-gated FET. Under the e-FET scheme, the quantum capacitance of the S-SWNTs, $\mathrm{C}_{\mathrm{SWNT}}$, and the electric double-layer (EDL) capacitance, $\mathrm{C}_{\mathrm{EDL}}$, forming around the $\mathrm{Ag} / \mathrm{AgCl}$ gate are connected in series, and therefore the $\mathrm{C}_{\mathrm{SWNTs}}$, being much lower than $\mathrm{C}_{\mathrm{EDL}}$, dominates the overall capacitance of the device. In addition, the $\mathrm{C}_{\mathrm{SWNTs}}$ is known to be much higher than the capacitance of back-gate dielectric $\left(\mathrm{SiO}_{2}\right), \mathrm{C}_{300 \mathrm{~nm}-\mathrm{SiO}_{2}}$ : that is, $\mathrm{C}_{300 \mathrm{~nm}-\mathrm{SiO}_{2}}$ $\ll \mathrm{C}_{\mathrm{SWNTS}} \ll \mathrm{C}_{\mathrm{EDL}}{ }^{38,39}$. Therefore, the operation voltage of the e-FET became significantly lower than that of the back-gated FET.

Figure $5 \mathrm{~b}$ shows that as the $\mathrm{pH}$ was increased, the transfer curve shifted toward a more positive voltage; that is, the $\mathrm{I}_{\mathrm{DS}}$ increased at a fixed gate voltage, $\mathrm{V}_{\mathrm{WG}}$. The $\mathrm{V}_{\mathrm{th}}$ values and the $\mathrm{I}_{\mathrm{DS}}$ levels at various $\mathrm{pH}$ values are plotted in Fig. $5 \mathrm{c}$. The $\mathrm{V}_{\text {th }}$ shift was observed to be about $30 \mathrm{mV} / \mathrm{pH}$ and the current response was about $6.7 \mu \mathrm{A} / \mathrm{pH}$. The $\mathrm{V}_{\text {th }}$ shift can be explained by the change of the surface charge of the p8GB\#1 phage in aqueous solution; that is, as the $\mathrm{pH}$ was increased, the phage became more negatively charged (Fig. 1c) and hence induced more positive carriers in the channels, shifting the threshold toward a more positive potential, as shown in Fig. 5b,c. Note that, as the $\mathrm{pH}$ was increased, the response of the e-FET began to increase more slowly at around $\mathrm{pH} \sim 7$. The flattening behaviour well correlates with the zeta potential dependence of the phage on $\mathrm{pH}$ in Fig. 1c, confirming the gate effect of the phage on S-SWNTs. In the nanomesh, the chemical functional groups of the biological template is in intimate contact with the S-SWNTs and therefore the phage serves as an effective gate for the S-SWNTs even in the high ionic strength of buffer solution. Note that the ionic strength of the $\mathrm{pH}$ buffer solution was high, at $10 \mathrm{mM}$. The 
(a)

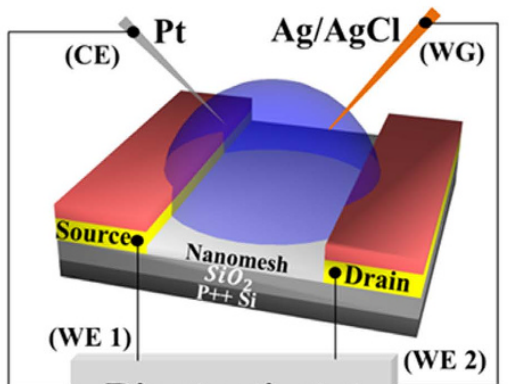

Bipotentiostat

(b)

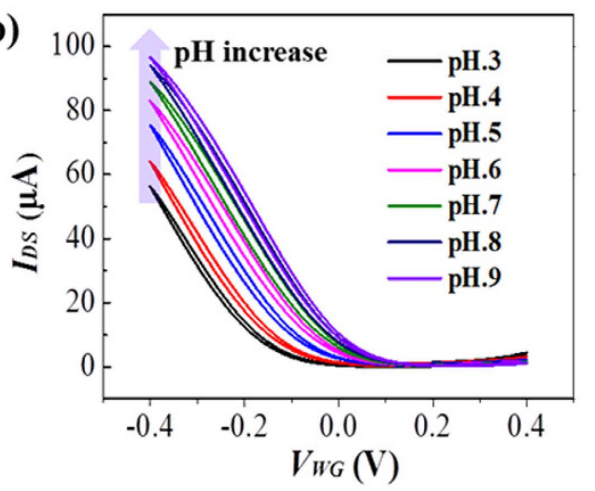

(c)

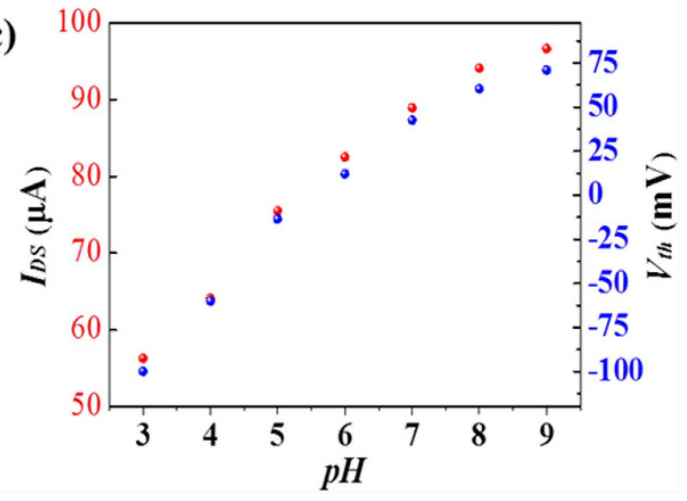

Figure 5. Semiconducting nanomesh-based e-FETs and the biological gate effect of the surface charge of the M13 phage. (a) A schematic illustration of the e-FET device and the configuration used to make the measurements. The Ag/ AgCl reference electrode serves as the water-based electrolyte gate (WG). (b) The transfer characteristics of the e-FET at various $\mathrm{pH}$ values. $\mathrm{V}_{\mathrm{DS}}=0.4 \mathrm{~V}$. (c) The dependence of the $\mathrm{I}_{\mathrm{DS}}$ and the $\mathrm{V}_{\text {th }}$ of the semiconducting nanomesh-based e-FET on $\mathrm{pH}$.

Debye length, $\lambda$, which describes the extent to which the electric field, $-\nabla \psi$, extends from the charged surface, decreases with increasing ionic strength (being a few $\mathrm{nm}$ in $10 \mathrm{mM}$ electrolyte) since the surface charge and the resultant electric field are readily screened by counter ions in solution ${ }^{33,41}$. Therefore, only when the sensing channel is within the Debye length, can any change of charge caused by a chemical or biological reaction or by a specific binding event be electrically detected in an effective manner ${ }^{42}$.

The biological gate effect of the biological phage material was further investigated using dopamine. Dopamine was chosen since it has an aromatic group that is expected to interact with the aromatic residue (W, tryptophan) on the surface of the body of the p8GB\#1 via $\pi-\pi$ interactions ${ }^{43}$; at the same time it is positively charged (pI 8.93) in physiological solution ( $\mathrm{pH} \sim 7.4)^{44}$ and therefore the binding of dopamine could reduce the effective charge density of the phage. Figure 6 a shows the transfer characteristics of the e-FET measured at various concentrations of dopamine in $10 \mathrm{mM}$ PBS buffer solution. The limit of detection (LOD, $\mathrm{S} / \mathrm{N}=3$ ) for the dopamine was estimated to be $\sim 80 \mathrm{pM}$. As the concentration of dopamine was increased, the $\mathrm{I}_{\mathrm{DS}}$ value was found to decrease. The change in current as the dopamine concentration was increased at a $\mathrm{V}_{\mathrm{WG}}$ of $-0.4 \mathrm{~V}$ is presented in Fig. $6 \mathrm{~b}$. Note that several studies have reported that dopamine introduced a p-doping effect on SWNTs, reduced graphene oxide (rGO), and CVD-graphene-based e-FETs, leading to an increase of $\mathrm{I}_{\mathrm{DS}}$ or positive shift of $\mathrm{V}_{\text {th }}{ }^{9,45}$. This behaviour is in contrast to our results where the $\mathrm{V}_{\text {th }}$ shifted toward a more negative voltage and the $\mathrm{I}_{\mathrm{DS}}$ decreased. It 
(a)

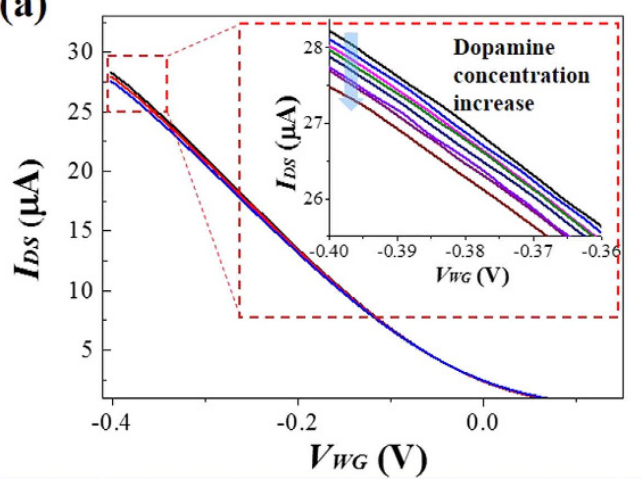

(b)

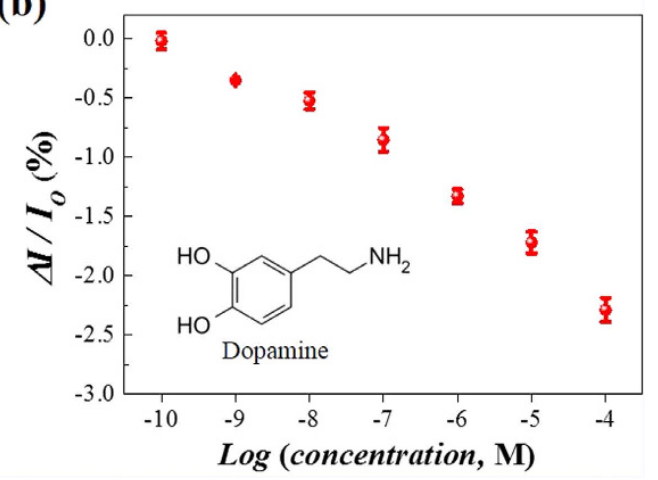

Figure 6. Electrochemical modulation of the semiconducting nanomesh-based e-FET by dopamine binding. (a) The transfer characteristics of the semiconducting nanomesh-based e-FET at various concentrations of dopamine in a biologically relevant solution (10 mM PBS buffer solution). Enlarged views of the transfer curves around $\mathrm{V}_{\mathrm{WG}} \sim-0.4$ are shown in the inset. (b) The dependence of the $\mathrm{I}_{\mathrm{DS}}$ on the concentration of dopamine. Each data point is the mean \pm s.d. from $\mathrm{n}=3$ devices.

seems that in our system the modulation of the gate effect of the phage by the binding of dopamine was more dominant than the doping effect of the adsorbed dopamine on the S-SWNTs, which is ascribed to the preferential binding of the aromatic molecules with the phage and the interaction of the majority of the S-SWNT surface with the bigger phage.

Selective detection of BPA with aptamer-conjugated nanomesh-based e-FET. The dominant gating effect of the biological template material on the nanostructured semiconducting channels further allows for additional functionality via modification of the template surface. We conjugated an aptamer, a single-stranded DNA or RNA oligonucleotides, onto the phage surface to specifically bind small molecules. In this study, we utilized a specific aptamer designed for bisphenol A (BPA) detection as previously described in literature ${ }^{46}$. We chose BPA since endocrine disruptors such as BPA disrupt the normal endocrine functions influencing sexual development and reproduction of humans and animals, and ultimately result in infertility and various cancers. BPA associated with the cell membrane can simulate physiological responses even at low BPA concentrations $(\sim 100 \mathrm{pM})^{46,47}$. The sequence of ssDNA aptamer and its schematic structure is presented in Fig. 7a. Figure $7 \mathrm{~b}$ shows the transfer characteristics of the anti-BPA aptamer-conjugated nanomesh-based e-FET upon addition of various concentrations of BPA. The limit of detection ( $\mathrm{LOD}, \mathrm{S} / \mathrm{N}=3$ ) for the BPA was $\sim 0.4 \mathrm{ppt}$. The $\mathrm{I}_{\mathrm{DS}}$ linearly decreases up to $0.1 \mathrm{ppm}$ level of BPA, and starts flattening after $1 \mathrm{ppm}$ of BPA. This could be explained by the decreased effective negative charge of the aptamer anchored on the M13 phage, thus reducing the effective field acting on the S-SWNT channels in intimate contact with the phage in the nanomesh ${ }^{47-50}$. It has been proposed that the specific aptamer that we employed in this study specifically bound BPA and the binding reduced the effective charges around the aptamer presumably via either conformational change of the negatively charged aptamers or the screening of the aptamer by the specifically bound BPA ${ }^{48-50}$. It is noted that this preferential binding-induced response of the e-FET is much lower than the $\mathrm{pH}$-induced current modulation shown in Fig. 5b,c. Unlike the $\mathrm{pH}$ induced biological gating effect where the $\mathrm{pH}$ directly changes the surface charge of the M13 phage and accordingly the effective field, the binding of biomolecules indirectly affect the field acting on the SWNTs, resulting in a relatively weak response compared to the $\mathrm{pH}$ change. However, owing to the intimate contact of the SWNTs with the phage, the e-FET could detect BPA in a buffer solution of a high ionic strength.

We found that the aptamer-conjugated semiconducting nanomesh did not significantly respond to dopamine, or other BPA analogues such as bisphenol B and bisphenol C (Fig. S8) that are not expected to preferentially bind to the anti-BPA aptamer. These results suggest that our approach provides a promising material platform for bio/ electronics interfaces.

\section{Discussion}

We have demonstrated a biologically templated assembly of a high quality semiconducting nanomesh. The exquisite assembly of the individual S-SWNTs and M13 phages can lead to the fabrication of high-performance FETs $\left(\mathrm{I}_{\mathrm{on}} / \mathrm{I}_{\mathrm{off}} \sim 10^{4}\right)$ without relying on lithographic, chemical or annealing processes. Demonstration of high performance FETs indicates that the S-SWNTs can be effectively dispersed in the nanomesh via hydrodynamic process in which bundle formation or defect generation is minimized. We also showed the biological gate effect of the biological template material, M13 phage, on the semiconducting nanomesh. The biological gate effect enabled controllable and selective electrochemical interface modulation of the electronic nanomaterials. The semiconducting nanomesh-based e-FETs could detect BPA at the concentration level of ppt. The nanomesh-e-FET-based sensors could have great potential for detecting analytes in a $\mathrm{pH}$ controlled buffer solution or physiological solutions or sensing biological reactions that can change the $\mathrm{pH}$ surrounding the nanomesh channels. Our work suggests a promising route to realizing high-performance macroscale semiconductor devices out of nanoscale semiconducting materials and emerging applications such as wearable health monitoring devices and controlled release and monitoring of therapeutic agents ${ }^{51,52}$. 
(a)

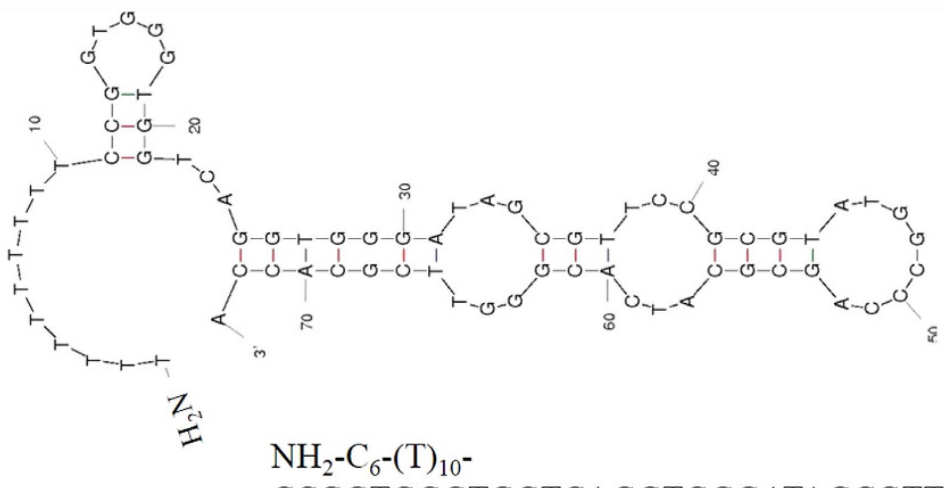

(b)

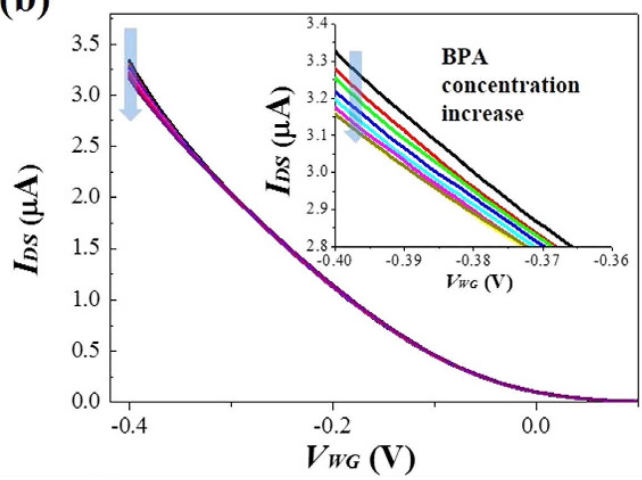

(c)

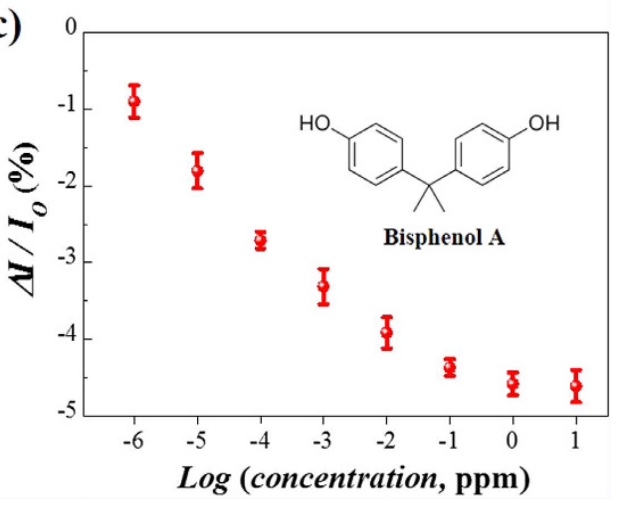

Figure 7. BPA detection with the aptamer-conjugated semiconducting nanomesh-based e-FET. (a) Single-stranded DNA aptamer structure for binding of bisphenol A (anti-BPA aptamer). (b) The transfer characteristics of the aptamer-conjugated nanomesh-based e-FET at various concentrations of BPA in a biologically relevant solution (10 mM PBS buffer solution). Enlarged views of the transfer curves around $\mathrm{V}_{\mathrm{WG}} \sim-0.4$ are shown in the inset. (c) The dependence of the $\mathrm{I}_{\mathrm{DS}}$ on the concentration of BPA. Each data point is the mean \pm s.d. from $n=3$ devices.

\section{Methods}

Biologically templated assembly of the semiconducting nanomesh. The semiconducting nanomesh was hydrodynamically assembled using a biological template material as shown in Fig. $1 \mathrm{a}^{27}$. Briefly, as-received 99\% semiconductor-enriched SWNT solution (IsoNanotubes-S-99\% from NanoIntegris Inc.) was first dialyzed against a sodium cholate solution (anionic surfactant, $2 \% \mathrm{w} / \mathrm{v}$ in deionized water) for two days using a dialysis membrane (SpectraLabs, product \#132706, MWCO 12,000 14,000 Daltons) with frequent buffer changes. This surfactant-exchange process is critical to effectively debundle and stabilize individual S-SWNTs. Unlike unsorted or mixed SWNTs, the as-received S-SWNTs are coated with surfactants used in the process of enriching or sorting S-SWNTs. This surfactant causes the agglomeration of S-SWNTs in the nanomesh. Proper replacement of it with sodium chlorate leads to high quality semiconducting nanomesh and high performance FETs. The S-SWNTs stabilized by the sodium cholate surfactant were mixed with the M13 phage showing strong binding affinity toward SWNTs on its body surface, p8GB\#1 phage, with various S-SWNT:p8GB\#1 molar ratios. A $5 \times 10^{11} / \mathrm{mL}$ number concentration of S-SWNTs was used to prepare a nanomesh with a 1:2 molar ratio of S-SWNT:p8GB\#1 (Supplementary equation). The mixed solution was then put into a dialysis membrane (SpectraLabs, product \#132700, MWCO 12,000 14,000 Daltons) and dialyzed against deionized water with frequent changing of the dialyzing solution. After about 30 hours, the dialyzed membrane bag was taken out to a container filled with water and then the dialysis membrane was removed, producing a large-area nanomesh film floating in water.

Characterizations of the assembled nanomesh. The Raman spectra for the S-SWNTs were obtained using a RENISHAW InVia Raman Microscope, using a $532 \mathrm{~nm}$ laser with a focal beam size of $2 \mu \mathrm{m}$. For the TEM and EF-TEM measurements, the nanomeshes were transferred to a TEM grid (QUANTIFOIL $2 \mu \mathrm{m}$ circular holes, TedPella Inc.) and dried at room temperature. TEM and EF-TEM were performed using a Quantum 966 of FEI Titan, operated at $300 \mathrm{kV}$. 
Zeta potential measurement. For measuring the zeta potential of p8GB\# 1 phage, seven different phosphate citrate buffer solutions ( $1 \mathrm{mM}, \mathrm{pH}$ values from 3 to 9 ) were prepared by mixing $150 \mu \mathrm{L}$ of p8GB\#1 phage $\left(1.4 \times 10^{14} \mathrm{phage} / \mathrm{ml}, \sim 4 \mathrm{mg} / \mathrm{ml}\right)$ with $600 \mu \mathrm{L}$ of $1 \mathrm{mM}$ phosphate citrate buffer. The zeta potential of $\mathrm{p} 8 \mathrm{~GB} \# 1$ phage in phosphate citrate buffer solution was measured using a Malvern Zetasizer Nano ZS.

Fabrication of semiconducting nanomesh-based FETs and e-FETs. Freestanding nanomesh was transferred onto a cleaned $\mathrm{SiO}_{2}(300 \mathrm{~nm}) / \mathrm{Si}$ substrate using a pre-patterned stencil mask. The transferred layer was left to dry in air, and then the stencil mask was lifted off to produce channels. Then, an additional $150 \mathrm{~nm}$ thick Au layer was deposited using the sputtering method to form source-drain contact electrodes. The length and the width of the channel were set to $200 \mu \mathrm{m}$ and $400 \mu \mathrm{m}$, respectively. The $\mathrm{SiO}_{2}$ served as the back-gate dielectric and the heavily doped Si substrate as the back-gate. The e-FET devices were fabricated by transferring the 1:2 molar ratio nanomesh onto interdigitated Au electrodes (Dropsens, IDEAU200). These electrodes served as the source and drain electrodes of the e-FET. The electrode gap (channel length) was $200 \mu \mathrm{m}$. The surface of the gold electrodes that was not covered by the nanomesh was passivated using cyanoacrylate adhesive. For the conjugation of the aptamers onto the nanomesh channel for the binding of bisphenol A (BPA), a specific aptamer ${ }^{46}$ with a modification of amine group at $5^{\prime}$ ends $\left(\mathrm{NH}_{2}-\mathrm{C}_{6}-(\mathrm{T})_{10}\right.$-CCGGTGGGTG GTCAGGTGGGATAGCGTTCCGCGTATGGCCCAGCGCATCACGGGTTCGCACCA, Bioneer Corporation, Daejeon, Korea) was synthesized and cross-linked onto the amine functional groups of the p8GB\#1 phage surface using a cross-linker (1\% glutaraldehyde). The device was carefully washed with DI water after cross-linking in order to remove physically absorbed aptamer molecules.

FET and e-FET measurements. Back-gated FETs were characterized using a physical property measurement system (PPMS, Quantum design). For the e-FET measurement, the source, drain and the water-based electrolyte gate (WG) potential were driven in a biological liquid electrolyte (10 mM PBS) using a bipotentiostat system (Autolab, PGSTAT 128N). Briefly, the source and drain electrodes were designated as two working electrodes (WE1 and WE2), the $\mathrm{Ag} / \mathrm{AgCl}$ reference electrode (RE) ( 2 mm dia. filled with $3 \mathrm{M} \mathrm{KCl}$ ) as the water-gated electrolyte gate electrode, and the additional Pt wire as the counter electrode (CE). The $\mathrm{I}_{\mathrm{DS}}-\mathrm{V}_{\mathrm{DS}}$ and $\mathrm{I}_{\mathrm{DS}}-\mathrm{V}_{\mathrm{WG}}$ measurements of the e-FET device were taken in a PBS buffer $(10 \mathrm{mM})$ at a scan rate of $10 \mathrm{mV} / \mathrm{s}$. The $\mathrm{pH}$-induced gating of the semiconducting nanomesh-based e-FET was analyzed in $10 \mathrm{mM}$ phosphate citrate buffer solution by testing various $\mathrm{pH}$ values $(3 \sim 9)$ at a fixed $\mathrm{V}_{\mathrm{DS}}$ of $0.4 \mathrm{~V}$ with a scan rate of $10 \mathrm{mV} / \mathrm{s}$. Dopamine solutions were prepared by dissolving dopamine hydrochloride (Sigma Aldrich) in $10 \mathrm{mM}$ PBS buffer solution, and the e-FET measurement (gate sweep from -0.4 to $0.4 \mathrm{~V}$ ) was carried out at a fixed $\mathrm{V}_{\mathrm{DS}}$ of $0.1 \mathrm{~V}$ to prevent electrochemical oxidation of dopamine molecules. Bisphenol A (BPA) solution was prepared by dissolving BPA in methanol to the concentration level of $100 \mathrm{ppm}$ first and then diluted by PBS solution to various concentrations. The e-FET measurement (gate sweep from -0.4 to $0.4 \mathrm{~V}$ ) was carried out at a fixed $\mathrm{V}_{\mathrm{DS}}$ of $0.2 \mathrm{~V}$.

\section{References}

1. Cui, Y., Wei, Q. Q., Park, H. K. \& Lieber, C. M. Nanowire Nanosensors for Highly Sensitive and Selective Detection of Biological and Chemical Species. Science 293, 1289-1292 (2001).

2. Takei, K. et al. Nanowire Active-Matrix Circuitry for Low-Voltage Macroscale Artificial Skin. Nat. Mater. 9, 821-826 (2010).

3. Duan, X. J. et al. Intracellular Recordings of Action Potentials by an Extracellular Nanoscale Field-Effect Transistor. Nat. Nanotechnol. 7, 174-179 (2012).

4. Lee, K. et al. In-situ Synthesis of Carbon Nanotube-Graphite Electronic Devices and Their Integrations onto Surfaces of Live Plants and Insects. Nano Lett. 14, 2647-2654 (2014).

5. Dvir, T., Timko, B. P., Kohane, D. S. \& Langer, R. Nanotechnological Strategies for Engineering Complex Tissues. Nat. Nanotechnol. 6, 13-22 (2011)

6. Park, J. U., Nam, S., Lee, M. S. \& Lieber, C. M. Synthesis of Monolithic Graphene-Graphite Integrated Electronics. Nat. Mater. 11, 120-125 (2012).

7. Kim, J. et al. Highly Transparent and Stretchable Field-Effect Transistor Sensors Using Graphene-Nanowire Hybrid Nanostructures. Adv. Mater. 27, 3292-3297 (2015).

8. Zhang, M. et al. High-Performance Dopamine Sensors Based on Whole-Graphene Solution-Gated Transistors. Adv. Funct. Mater. 24, 978-985 (2014).

9. He, Q. Y. et al. Centimeter-Long and Large-Scale Micropatterns of Reduced Graphene Oxide Films: Fabrication and Sensing Applications. ACS Nano 4, 3201-3208 (2010).

10. Cao, Q. \& Rogers, J. A. Ultrathin Films of Single-Walled Carbon Nanotubes for Electronics and Sensors: A Review of Fundamental and Applied Aspects. Adv. Mater. 21, 29-53 (2009).

11. Park, S., Vosguerichian, M. \& Bao, Z. A. A Review of Fabrication and Applications of Carbon Nanotube Film-Based Flexible Electronics. Nanoscale 5, 1727-1752 (2013).

12. Wong, W. S. \& Salleo, A. Editors, Flexible Electronics: Materials and Applications (Springer, 2009).

13. Kim, K. et al. Stretchable and Transparent Electrodes Based on In-Plane Structures. Nanoscale 7, 14577-14594 (2015).

14. An, B. W. et al. Stretchable, Transparent Electrodes as Wearable Heaters Using Nanotrough Networks of Metallic Glasses with Superior Mechanical Properties and Thermal Stability. Nano Lett. 16, 471-478 (2016).

15. Mann, S. Molecular Recognition in Biomineralization. Nature 332(6160), 119-124 (1988)

16. Sarikaya, M., Tamerler, C., Jen, A. K. Y., Schulten, K. \& Baneyx, F. Molecular Biomimetics: Nanotechnology through Biology. Nat. Mater. 2, 577-585 (2003).

17. Whaley, S. R., English, D. S., Hu, E. L., Barbara, P. F. \& Belcher, A. M. Selection of Peptides with Semiconductor Binding Specificity for Directed Nanocrystal Assembly. Nature 405, 665-668 (2000).

18. Baneyx, F. \& Schwartz, D. T. Selection and Analysis of Solid-Binding Peptides. Curr. Opin. Biotech 18, 312-317 (2007).

19. Han, T. H. et al. Peptide/Graphene Hybrid Assembly into Core/Shell Nanowires. Adv. Mater. 22, 2060-2064 (2010).

20. Han, S. P., Maune, H. T., Barish, R. D., Bockrath, M. \& Goddard, W. A. DNA-Linker-Induced Surface Assembly of Ultra Dense Parallel Single Walled Carbon Nanotube Arrays. Nano Lett. 12, 1129-1135 (2012).

21. Zheng, M. et al. DNA-Assisted Dispersion and Separation of Carbon Nanotubes. Nat. Mater. 2, 338-342 (2003).

22. Laaksonen, P. et al. Interfacial Engineering by Proteins: Exfoliation and Functionalization of Graphene by Hydrophobins. Angew. Chem. Int. Edit. 49, 4946-4949 (2010).

23. Wang, S. Q. et al. Peptides with Selective Affinity for Carbon Nanotubes. Nat. Mater. 2, 196-200 (2003). 
24. Reiss, B. D., Bai, G. R., Auciello, O., Ocola, L. E. \& Firestone, M. A. Identification of Peptides for the Surface Functionalization of Perovskite Ferroelectrics. Appl. Phys. Lett. 88, 083903 (2006).

25. Cui, Y. et al. Chemical Functionalization of Graphene Enabled by Phage Displayed Peptides. Nano Lett. 10, 4559-4565 (2010).

26. Khatayevich, D., So, C. R., Hayamizu, Y., Gresswell, C. \& Sarikaya, M. Controlling the Surface Chemistry of Graphite by Engineered Self-Assembled Peptides. Langmuir 28, 8589-8593 (2012).

27. Lee, K. Y. et al. Hydrodynamic Assembly of Conductive Nanomesh of Single-Walled Carbon Nanotubes Using Biological Glue. Adv . Mater. 27, 922-928 (2015).

28. Ju, S. et al. Single-Carbon Discrimination by Selected Peptides for Individual Detection of Volatile Organic Compounds. Sci. Rep. 5, 9196 (2015).

29. Lee, Y. J. et al. Fabricating Genetically Engineered High-Power Lithium-Ion Batteries Using Multiple Virus Genes. Science 324, 1051-1055 (2009).

30. Yi, H. J. et al. M13 Phage-Functionalized Single-Walled Carbon Nanotubes As Nanoprobes for Second Near-Infrared Window Fluorescence Imaging of Targeted Tumors. Nano Lett. 12, 1176-1183 (2012).

31. Lee, S. W., Mao, C. B., Flynn, C. E. \& Belcher, A. M. Ordering of Quantum Dots Using Genetically Engineered Viruses. Science 296, 892-895 (2002).

32. Dang, X. N. et al. Virus-Templated Self-Assembled Single-Walled Carbon Nanotubes for Highly Efficient Electron Collection in Photovoltaic Devices. Nat. Nanotechnol. 6, 377-384 (2011).

33. Hiemenz, P. C. \& Rajagopalan, R. Principles of Colloid and Scurface Chemistry (CRC press, Boca Raton, 1997).

34. Loiseau, A., Petit, P., Roche, S., Launois, P. \& Salvetat, J.-P. Understanding Carbon Nanotubes (Springer, Berlin, 2006).

35. Kim, W. et al. Hysteresis Caused by Water Molecules in Carbon Nanotube Field-Effect Transistors. Nano Lett. 3, 193-198 (2003).

36. Lee, J. S. et al. Origin of Gate Hysteresis in Carbon Nanotube Field-Effect Transistors. J. Phys. Chem. C 111, 12504-12507 (2007).

37. Jin, S. H. et al. Sources of Hysteresis in Carbon Nanotube Field-Effect Transistors and Their Elimination Via Methylsiloxane Encapsulants and Optimized Growth Procedures. Adv. Funct. Mater. 22, 2276-2284 (2012).

38. Rosenblatt, S. et al. High Performance Electrolyte Gated Carbon Nanotube Transistors. Nano Lett. 2, 869-872 (2002).

39. Ozel, T., Gaur, A., Rogers, J. A. \& Shim, M. Polymer Electrolyte Gating of Carbon Nanotube Network Transistors. Nano Lett. 5, 905-911 (2005)

40. Lee, E. H., Lee, S. W. \& Saraf, R. F. Noninvasive Measurement of Membrane Potential Modulation in Microorganisms: Photosynthesis in Green Algae. ACS Nano 8, 780-786 (2014).

41. Kostiainen, M. A. et al. Electrostatic Assembly of Binary Nanoparticle Superlattices Using Protein Cages. Nat. Nanotechnol. 8, 52-56 (2013).

42. Palazzo, G. et al. Detection Beyond Debye's Length with an Electrolyte-Gated Organic Field-Effect Transistor. Adv. Mater. 27, 911-916 (2015).

43. So, C. R. et al. Controlling Self-Assembly of Engineered Peptides on Graphite by Rational Mutation. ACS Nano 6, 1648-1656 (2012).

44. Lee, S. W., Lopez, J. \& Saraf, R. F. Fabrication and Properties of Redox Ion Doped Few Monolayer Thick Polyelectrolyte Film for Electrochemical Biosensors at High Sensitivity and Specificity. Electroanal. 25, 1557-1566 (2013).

45. Li, W. S. et al. High-Quality, Highly Concentrated Semiconducting Single-Wall Carbon Nanotubes for Use in Field Effect Transistors and Biosensors. ACS Nano 7, 6831-6839 (2013).

46. Jo, M. et al. Development of Single-Stranded DNA Aptamers for Specific Bisphenol A Detection. Oligonucleotides 21, 85-91 (2011).

47. Kuang, H. et al. Asymmetric Plasmonic Aptasensor for Sensitive Detection of Bisphenol A. ACS Appl. Mater. Interfaces 6, 364-369 (2014).

48. Xu, J. Y. et al. Colorimetric Method for Determination of Bisphenol A Based on Aptamer-Mediated Aggregation of Positively Charged Gold Nanoparticles. Microchim. Acta 182, 2131-2138 (2015).

49. Zhu, Y. Y. et al. Building an Aptamer/Graphene Oxide FRET Biosensor for One-Step Detection of Bisphenol A. ACS Appl. Mater. Interfaces 7, 7492-7496 (2015).

50. Kim, S. G., Lee, J. S., Jun, J., Shin, D. H. \& Jang, J. Ultrasensitive Bisphenol A. Field-Effect Transistor Sensor Using an AptamerModified Multichannel Carbon Nanofiber Transducer. ACS Appl. Mater. Interfaces 8, 6602-6610 (2016).

51. Huang, S. C. J. et al. Carbon Nanotube Transistor Controlled by a Biological Ion Pump Gate. Nano Lett. 10, 1812-1816 (2010).

52. Nehra, A. \& Singh, K. P. Current Trends in Nanomaterial Embedded Field Effect Transistor-Based Biosensor. Biosens. Bioelectron. 74, 731-743 (2015)

\section{Acknowledgements}

This work was supported by KIST through the Institutional Program (2E26380, 2V04930). This research was also in part supported by the Bio \& Medical Technology Development Program (Grant No. NRF2015M3A9E2030105) and the Basic Science Research Program (Grant No. NRF-2014R1A1A3051952) of the National Research Foundation (NRF) funded by the Ministry of Science, ICT \& Future Planning. S.W.L. is grateful for the research fellowship from National Research Council of Science \& Technology (NST) of Korea. We would like to thank Dr. Do Kyung Hwang for helpful discussion, Mr. Kun Soo Song for the SEM analysis, and Ms. Min Kyung Cho for the TEM and EF-TEM analysis in the Advanced Analysis Center at KIST.

\section{Author Contributions}

H.-H.B., S.-W.L., W.K. and H.Y. conceived the idea and, together with E.-H.L. designed the experiments. H.-H.B., S.-W.L. and E.-H.L. performed the experiments. All authors discussed the results, co-wrote the manuscript and commented on the manuscript.

\section{Additional Information}

Supplementary information accompanies this paper at http://www.nature.com/srep

Competing financial interests: The authors declare no competing financial interests.

How to cite this article: Byeon, H.-H. et al. Biologically templated assembly of hybrid semiconducting nanomesh for high performance field effect transistors and sensors. Sci. Rep. 6, 35591; doi: 10.1038/srep35591 (2016).

This work is licensed under a Creative Commons Attribution 4.0 International License. The images or other third party material in this article are included in the article's Creative Commons license, unless indicated otherwise in the credit line; if the material is not included under the Creative Commons license, users will need to obtain permission from the license holder to reproduce the material. To view a copy of this license, visit http://creativecommons.org/licenses/by/4.0/

(c) The Author(s) 2016 